\title{
Blocking the alternative complement pathway
}

The complement system is a key component of the innate immune system, which constitutes an effective first line of defence against invading pathogens. However, inappropriate or uncontrolled complement activation can be deleterious and has been implicated in various diseases. Now, writing in Nature Chemical Biology, Anderson and colleagues report the identification of a small-molecule

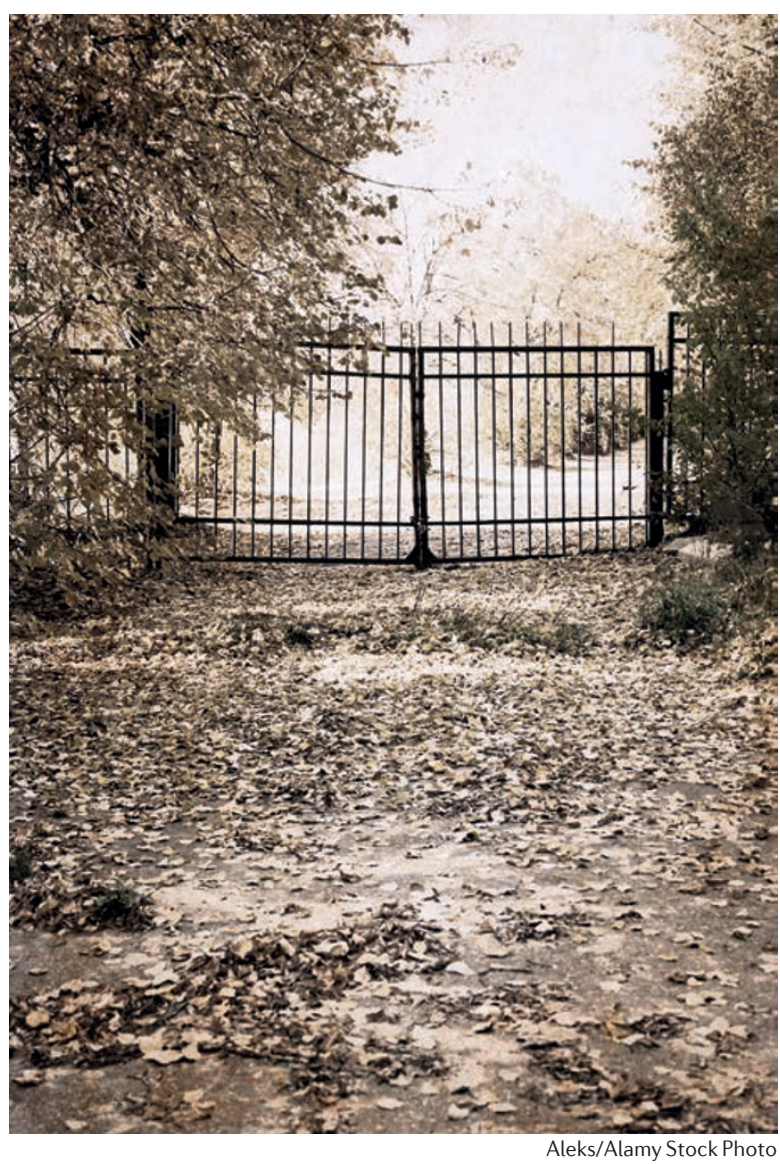

inhibitor of complement factor D (FD), which blocked activity of the alternative pathway (AP) of complement activation in mice and prevented lysis of erythrocytes from individuals with paroxysmal nocturnal haemoglobinuria (PNH).

The AP is one of three pathways that converge at the proteolytic cleavage of complement 3 (C3), which generates the activation fragments $\mathrm{C} 3 \mathrm{a}$ and $\mathrm{C} 3 \mathrm{~b}$; it also acts as an amplification loop for the other two pathways (classical and lectin). FD is the rate-limiting enzyme of the AP and has emerged as a promising therapeutic target for complement-mediated diseases. Although an antigen-binding fragment capable of neutralizing FD activity is in clinical trials for the treatment of atrophic age-related macular degeneration (AMD), its use is limited to intravitreal injection. With this limitation in mind, Anderson and colleagues set out to identify small-molecule inhibitors of FD.

Using a combination of structure-based drug design and fragment-based screening, followed by extensive chemical optimization, the authors generated the 6 -aza-indazole compound 6 as a highly potent and selective inhibitor of human FD. The inhibitor effectively blocked both AP-mediated haemolysis in human serum and AP-induced membrane attack complex (MAC) formation in human whole blood.

In vitro, compound 6 blocked C3 cleavage in the serum of mice expressing human FD (human-FD knock-in (KI) mice), but not in the serum of wild-type mice. In a mouse model of systemic FD overactivity (such as occurs in AMD), in which lipopolysaccharide was injected into human-FD KI mice, orally administered compound 6 dose-dependently inhibited complement activation in both plasma and ocular tissue in vivo.

Finally, the FD inhibitor was shown to prevent C3-dependent opsonization; it inhibited C3 deposition on, and lysis of, erythrocytes from individuals with $\mathrm{PNH}$ - a rare, acquired, life-threatening disease of the blood, which is characterized by destruction of red blood cells mediated by the complement system. By contrast, the anti-C5 monoclonal antibody eculizumab (the current standard of treatment for $\mathrm{PNH}$ ) blocked haemolysis but generated erythrocytes that were extensively coated with C3 fragments, which are commonly observed in eculizumab-treated patients with $\mathrm{PNH}$ and are susceptible to extravascular haemolysis in the spleen and liver.

Taken together, these data support the potential use of small-molecule inhibitors of FD to target the AP in the treatment of complement-related diseases, for which therapies are currently limited.

Sarah Crunkhorn

ORIGINAL ARTICLE Maibaum, J. et al. Smallmolecule factor $D$ inhibitors targeting the alternative complement pathway. Nat. Chem. Biol. http://dx.doi:10.1038/nchembio.2208 (2016) FURTHER READING Morgan, B. P. \& Harris, C. L. Complement, a target for therapy in inflammatory and degenerative diseases. Nat. Rev. Drug Discov. 14, 857-877 (2015) 